\title{
ON THE ELASTOPLASTIC STABILITY PROBLEM OF THE THIN ROUND CYLINDRICAL SHELLS SUBJECTED TO COMPLEX LOADING PROCESSES WITH THE VARIOUS KINEMATIC BOUNDARY CONDITIONS
}

\author{
DAO VAN DUNG \\ Hanoi National University
}

\begin{abstract}
In this paper, the elastoplastic stability of cylindrical shells simultaneously subjected to compression force along the generatrix and external pressure has been presented. Two types of considered kinematic boundary conditions are simply supported and clamped at the butt-ends. The expressions for determining the critical forces by using the Bubnov-Galerkin method [3] have been established. The sufficient condition of extremum for a long cylindrical shell also is considered. Some results of numerical calculation have been also given and discussed.
\end{abstract}

\section{Stability problem}

Let's consider a thin round cylindrical shell of length $L$, radius $R$ and thickness $h$. We choose a orthogonal coordinate system $O x_{1} x_{2} x_{3}$ so that the axis $O x_{1}$ belonging to the middle surface and lying along the generatrix of the shell, $x_{2}=R \theta_{1}$ with $\theta_{1}$-the angle of circular arc and $x_{3}$ in the direction of the normal to the middle surface.

Assume that a material of shell is incompressible and shell is subjected to the compression force $p(t)$ along the generatrix and external pressure $q_{1}(t)$ which depend arbitrarily on a loading parameter $t$. One of the main aims of the stability problem is to find the moment $t_{*}$ when the instability of the structure happens and respectively the critical loads $p^{*}=p\left(t_{*}\right), q_{1}^{*}=q_{1}\left(t_{*}\right)$. Suppose that the unloading does not happen in the structure. We use the criterion of bifurcation of equilibrium state to investigate the proposed problem.

An investigation of the elastoplastic stability problem is always made two parts: prebuckling process and post-buckling process.

\subsection{Pre-buckling process}

Suppose that at any moment $t$ there exists a membrane plane stress state in the cylindrical shell

$$
\sigma_{11}=-p(t) \equiv-p ; \quad \sigma_{22}=-q_{1}(t) \frac{R}{h} \equiv-q(t) \equiv-q, \quad \sigma_{12}=\sigma_{13}=\sigma_{23}=\sigma_{33}=0 .
$$

Thus

$$
\sigma=\frac{\sigma_{11}+\sigma_{22}}{3}=-\frac{p+q}{3}, \quad \sigma_{u}=\sqrt{\sigma_{11}^{2}-\sigma_{11} \sigma_{22}+\sigma_{22}^{2}}=\sqrt{p^{2}-p q+q^{2}} .
$$


Components of the strain velocity tensor determined according to the theory of elastoplastic processes [1] are of the form

$$
\begin{aligned}
& \dot{\varepsilon}_{11}=\frac{1}{N}\left(-\dot{p}+\frac{1}{2} \dot{q}\right)-Q(s, t)\left(p-\frac{1}{2} q\right), \\
& \dot{\varepsilon}_{22}=\frac{1}{N}\left(-\dot{q}+\frac{1}{2} \dot{p}\right)-Q(s, t)\left(q-\frac{1}{2} p\right), \\
& \dot{\varepsilon}_{33}=-\left(\dot{\varepsilon}_{11}+\dot{\varepsilon}_{22}\right), \quad \dot{\varepsilon}_{12}=\dot{\varepsilon}_{13}=\dot{\varepsilon}_{23}=0,
\end{aligned}
$$

where

$$
Q(s, t)=\left(\frac{1}{\phi^{\prime}}-\frac{1}{N}\right) \frac{p \dot{p}+q \dot{q}-\frac{1}{2} p \dot{q}-\frac{1}{2} \dot{p} q}{p^{2}-p q+q^{2}}, \quad \phi^{\prime}=\phi^{\prime}(s) ; \quad N=\frac{\sigma_{u}}{s} .
$$

The arc-length of the strain trajectory is given respectively by the formula

$$
\frac{d s}{d t}=\frac{2}{\sqrt{3}}\left(\dot{\varepsilon}_{11}^{2}+\dot{\varepsilon}_{11} \dot{\varepsilon}_{22}+\dot{\varepsilon}_{22}^{2}\right)^{1 / 2} \equiv F(s, t) .
$$

So, we can determine, from equations $(1.1) \div(1.3)$ associating with boundary conditions and the equilibrium equations, stress and strain states at any point $M$ in the cylindrical shell at any moment of the prebuckling process.

\subsection{Post-buckling process}

As shown in $[1,4]$, the system of stability equations of the cylindrical shell is written in the form

$$
\begin{aligned}
& \beta_{1} \frac{\partial^{4} \varphi}{\partial x_{1}^{4}}+\beta_{3} \frac{\partial^{4} \varphi}{\partial x_{1}^{2} \partial x_{2}^{2}}+\beta_{5} \frac{\partial^{4} \varphi}{\partial x_{2}^{4}}+\frac{N}{R} \frac{\partial^{2} \delta w}{\partial x_{1}^{2}}=0 \\
& \alpha_{1} \frac{\partial^{4} \delta w}{\partial x_{1}^{4}}+\alpha_{3} \frac{\partial^{4} \delta w}{\partial x_{1}^{2} \partial x_{2}^{2}}+\alpha_{5} \frac{\partial^{4} \delta w}{\partial x_{2}^{2}}+\frac{9}{N h^{2}}\left(p \frac{\partial^{2} \delta w}{\partial x_{1}^{2}}+q \frac{\partial^{2} \delta w}{\partial x_{2}^{2}}-\frac{1}{R} \frac{\partial^{2} \varphi}{\partial x_{1}^{2}}\right)=0
\end{aligned}
$$

where the coefficients $\alpha_{i}, \beta_{i}(i=1,3,5)$ are calculated as follows

$$
\begin{aligned}
& \beta_{1}=1+\frac{1}{4}\left(\frac{N}{\phi^{\prime}}-1\right) \frac{(2 q-p)^{2}}{p^{2}-p q+q^{2}}, \\
& \beta_{3}=2+\frac{1}{2}\left(\frac{N}{\phi^{\prime}}-1\right) \frac{(2 p-q)(2 q-p)}{p^{2}-p q+q^{2}}, \\
& \beta_{5}=1+\frac{1}{4}\left(\frac{N}{\phi^{\prime}}-1\right) \frac{(2 p-q)^{2}}{p^{2}-p q+q^{2}}, \\
& \alpha_{1}=1-\frac{3}{4}\left(1-\frac{\phi^{\prime}}{N}\right) \frac{p^{2}}{p^{2}-p q+q^{2}}, \\
& \alpha_{3}=2-\frac{3}{2}\left(1-\frac{\phi^{\prime}}{N}\right) \frac{p q}{p^{2}-p q+q^{2}}, \\
& \alpha_{5}=1-\frac{3}{4}\left(1-\frac{\phi^{\prime}}{N}\right) \frac{q^{2}}{p^{2}-p q+q^{2}} .
\end{aligned}
$$


In order to solve the stability problem of the cylindrical shell, we consider two types of kinematic boundary conditions following

* the shell is simply supported at the planes $x_{1}=0$ and $x_{1}=L$

* the shell is clamped at the planes $x_{1}=0$ and $\dot{x}_{1}=L$.

Hereafter we will study the solution of these two stability problems

\section{Solving the elastoplastic stability problem of simply sup- ported cylindrical shell}

We find the solution $\delta w$ which satisfies the mentioned boundary conditions in the form

$$
\delta w=\sum_{m=1}^{M} \sum_{n=1}^{M} A_{m n} \sin \frac{m \pi x_{1}}{L} \sin \frac{n x_{2}}{R} .
$$

It is easy to see that the system of functions $\delta w_{m n}=\sin \frac{m \pi x_{1}}{L} \sin \frac{n x_{2}}{R}$ is linearly independence.

Substituting this expression into (1.4), we can obtain the particular solution $\varphi$ as follows

$$
\varphi=\sum_{m=1}^{M} \sum_{n=1}^{M} B_{m n} \sin \frac{m \pi x_{1}}{L} \sin \frac{n x_{2}}{R}
$$

where

$$
B_{m n}=\frac{N}{R}\left(\frac{m \pi}{L}\right)^{2} A_{m n}\left[\beta_{1}\left(\frac{m \pi}{L}\right)^{4}+\beta_{3}\left(\frac{m \pi}{L}\right)^{2}\left(\frac{n}{R}\right)^{2}+\beta_{5}\left(\frac{n}{R}\right)^{4}\right]^{-1} .
$$

Now we pass to find the expression determining the critical forces by the Bubnov-Galerkin method. For doing that, one need to realize the following steps:

a) Substituting the expressions of $\delta w$ and $\varphi$ from (2.1), (2.2) into (1.5).

b) Multiplying both sides of that stability equation by

$$
\delta w_{i j}=\sin \frac{i \pi x_{1}}{L} \sin \frac{j x_{2}}{R} .
$$

c) Integrating the received just equation following $x_{1}$ and $x_{2}$.

Finally, we reach

$$
\begin{aligned}
\int_{0}^{L} \int_{0}^{2 \pi R}\left\{\alpha_{1} \frac{\partial^{4} \delta w}{\partial x_{1}^{4}}+\alpha_{3} \frac{\partial^{4} \delta w}{\partial x_{1}^{2} \partial x_{2}^{2}}+\alpha_{5} \frac{\partial^{4} \delta w}{\partial x_{2}^{4}}+\frac{9}{h^{2} N}\left(p \frac{\partial^{2} \delta w}{\partial x_{1}^{2}}+q \frac{\partial^{2} \delta w}{\partial x_{2}^{2}}\right)\right. \\
\left.-\frac{9}{h^{2} N R} \frac{\partial^{2} \varphi}{\partial x_{1}^{2}}\right\} \sin \frac{i \pi x_{1}}{L} \sin \frac{j x_{2}}{R} d x_{1} d x_{2}=0 \quad(i, j=1,2, \ldots, M) .
\end{aligned}
$$

For taking this integral, it needs to use the result

$$
\int_{0}^{L} \int_{0}^{2 \pi R} \sin \frac{m \pi x_{1}}{L} \sin \frac{i \pi x_{1}}{L} \sin \frac{n x_{2}}{R} \sin \frac{j x_{2}}{R} d x_{1} d x_{2}= \begin{cases}0 & \text { with } m \neq i, n \neq j \\ \frac{1}{2} \pi R L & \text { with } m=i, n=j .\end{cases}
$$


After series of calculations, the relation (2.4) gives us

$$
\begin{aligned}
& \frac{\pi R L}{2}\left\{\alpha_{1}\left(\frac{m \pi}{L}\right)^{4}+\alpha_{3}\left(\frac{m \pi}{L}\right)^{2}\left(\frac{n}{R}\right)^{2}+\alpha_{5}\left(\frac{n}{R}\right)^{4}-\frac{9}{h^{2} N}\left[p\left(\frac{m \pi}{L}\right)^{2}+q\left(\frac{n}{R}\right)^{2}\right]\right. \\
& \left.+\frac{9}{h^{2} R^{2}}\left(\frac{m \pi}{L}\right)^{4}\left[\beta_{1}\left(\frac{m \pi}{L}\right)^{4}+\beta_{3}\left(\frac{m \pi}{L}\right)^{2}\left(\frac{n}{R}\right)^{2}+\beta_{5}\left(\frac{n}{R}\right)^{4}\right]^{-1}\right\} A_{m n}=0 .
\end{aligned}
$$

Taking into account the existence of non-trivial solution i.e. $A_{m n} \neq 0$, we receive the expression for determining critical loads

$$
\begin{aligned}
p\left(\frac{m \pi}{L}\right)^{2}+q\left(\frac{n}{R}\right)^{2}= & \frac{h^{2} N}{9}\left\{\alpha_{1}\left(\frac{m \pi}{L}\right)^{4}+\alpha_{3}\left(\frac{m \pi}{L}\right)^{2}\left(\frac{n}{R}\right)^{2}+\alpha_{5}\left(\frac{n}{R}\right)^{4}\right\} \\
& +\frac{N}{R^{2}}\left(\frac{m \pi}{L}\right)^{4}\left\{\beta_{1}\left(\frac{m \pi}{L}\right)^{4}+\beta_{3}\left(\frac{m \pi}{L}\right)^{2}\left(\frac{n}{R}\right)^{2}+\beta_{5}\left(\frac{n}{R}\right)^{4}\right\}^{-1} .
\end{aligned}
$$

Noticing that the relation (2.6) coincides with one established by another method in $[1,4]$. By putting $\psi=n^{2}, \theta=\left(\frac{m \pi R}{n L}\right)^{2}, i=\frac{3 R}{h}$, the relation (2.6) is written in the form

$$
i^{2}=\frac{N \psi^{2}\left(\alpha_{1} \theta+\alpha_{3}+\frac{\alpha_{5}}{\theta}\right)\left(\beta_{1} \theta+\beta_{3}+\frac{\beta_{5}}{\theta}\right)}{\left(p+\frac{q}{\theta}\right)\left(\beta_{1} \theta+\beta_{3}+\frac{\beta_{5}}{\theta}\right) \psi-N} .
$$

Minimizing this expression, i.e. $\frac{\partial i^{2}}{\partial \psi}=0, \frac{\partial i^{2}}{\partial \theta}=0$, after some calculations we get

$$
\begin{gathered}
\psi=\frac{2 N}{\left(p+\frac{q}{\theta}\right)\left(\beta_{1} \theta+\beta_{3}+\frac{\beta_{5}}{\theta}\right)} . \\
\left(\alpha_{1}-\frac{\alpha_{5}}{\theta^{2}}\right)\left(\beta_{1} \theta+\beta_{3}+\frac{\beta_{5}}{\theta}\right)-\left(\beta_{1}-\frac{\beta_{5}}{\theta^{2}}\right)\left(\alpha_{1} \theta+\alpha_{3}+\frac{\alpha_{5}}{\theta}\right)+ \\
\frac{2 q}{\theta^{2}\left(p+\frac{q}{\theta}\right)}\left(\alpha_{1} \theta+\alpha_{3}+\frac{\alpha_{5}}{\theta}\right)\left(\beta_{1} \theta+\beta_{3}+\frac{\beta_{5}}{\theta}\right)=0 .
\end{gathered}
$$

Substituting the values (2.8) and (2.9) into (2.7) we have

$$
\begin{aligned}
i^{2} & =\frac{4 N^{2} \theta^{2}}{(p \theta+q)^{2}}\left\{\left[1-\frac{3}{4}\left(1-\frac{\phi^{\prime}}{N}\right) \frac{p^{2}}{p^{2}-p q+q^{2}}\right] \theta^{2}+\left[2-\frac{3}{2}\left(1-\frac{\phi^{\prime}}{N}\right) \frac{p q}{p^{2}-p q+q^{2}}\right] \theta+\right. \\
& \left.1-\frac{3}{4}\left(1-\frac{\phi^{\prime}}{N}\right) \frac{q^{2}}{p^{2}-p q+q^{2}}\right\} \cdot\left\{\left[1+\frac{1}{4}\left(\frac{N}{\phi^{\prime}}-1\right) \frac{(2 q-p)^{2}}{p^{2}-p q+q^{2}}\right] \theta^{2}+\right. \\
& {\left.\left[2+\frac{1}{2}\left(\frac{N}{\phi^{\prime}}-1\right) \frac{(2 q-p)(2 p-q)}{p^{2}-p q+q^{2}}\right] \theta+1+\frac{1}{4}\left(\frac{N}{\phi^{\prime}}-1\right) \frac{(2 p-q)^{2}}{p^{2}-p q+q^{2}}\right\}^{-1} . }
\end{aligned}
$$

where $\theta$ is a solution of the equation (2.9).

Applying the loading parameter method [1], we solve simultaneously the equation (1.3) and (2.10). After finding the critical value $t^{*}$, we can determine the critical forces as follows

$$
p^{*}=p\left(t^{*}\right), \quad q^{*}=q\left(t^{*}\right)
$$


For long cylindrical shells, i.e. $\psi=1, \theta \ll 1$, see [2], we deduce from (2.7)

$$
i^{2}=\frac{N \alpha_{5} \beta_{5}}{(p \theta+q) \beta_{5}-N \theta^{2}} .
$$

Minimizing the expression of $i^{2}$, i.e. $\frac{\partial i^{2}}{\partial \theta}=0$, gives us

$$
\theta=\frac{p \beta_{5}}{2 N}=\theta_{*}
$$

Now consider the sufficient condition of extremum [5]

$$
\left.\frac{\partial^{2} i^{2}}{\partial \theta^{2}}\right|_{\theta=\theta_{*}}=\frac{2 \alpha_{5} \beta_{5} N^{2}}{\left(\frac{p^{2} \beta_{5}^{2}}{4 N}+q \beta_{5}\right)^{2}} .
$$

Because

$$
\begin{aligned}
& \alpha_{5}=1-\frac{3}{4}\left(1-\frac{\phi^{\prime}}{N}\right) \frac{q^{2}}{\sigma_{u}^{2}}=\frac{(2 p-q)^{2}}{4 \sigma_{u}^{2}}+\frac{3}{4} \frac{\phi^{\prime}}{N} \frac{q^{2}}{\sigma_{u}^{2}}>0, \\
& \beta_{5}=1+\frac{1}{4}\left(\frac{N}{\phi^{\prime}}-1\right) \frac{(2 p-q)^{2}}{\sigma_{u}^{2}}=\frac{3 q^{2}}{4 \sigma_{u}^{2}}+\frac{1}{4} \frac{N(2 p-q)^{2}}{\phi^{\prime} \sigma_{u}^{2}}>0 .
\end{aligned}
$$

So $\left.\frac{\partial^{2} i^{2}}{\partial \theta^{2}}\right|_{\theta=\theta_{*}}>0$, the sufficient condition of minimum is satisfied.

Substituting the values of $\alpha_{5}, \beta_{5}$ and $\theta=\theta_{*}$ into (2.11) we obtain

$$
i^{2}=\frac{4 N^{2}\left[1-\frac{3}{4}\left(1-\frac{\phi^{\prime}}{N}\right) \frac{q^{2}}{p^{2}-p q+q^{2}}\right]}{p^{2}\left[1+\frac{1}{4}\left(\frac{N}{\phi^{\prime}}-1\right) \frac{(2 p-q)^{2}}{p^{2}-p q+q^{2}}\right]+4 N q} .
$$

\section{Solving the elastoplastic stability problem of clamped cylin- drical shell}

The kinematic boundary conditions of the clamped shell at the planes $x_{1}=0$ and $x_{1}=L$ are satisfied completely by choosing

$$
\delta w=\sum_{m=1}^{M} \sum_{n=1}^{M} D_{m n}\left(1-\cos \frac{2 m \pi x_{1}}{L}\right) \sin \frac{n x_{2}}{R} .
$$

Using the expression of $\delta w$ and the equation (1.4) we can find the particular solution $\varphi$ in the form

$$
\varphi=\sum_{m=1}^{M} \sum_{n=1}^{M} E_{m n} \cos \frac{2 m \pi x_{1}}{L} \sin \frac{n x_{2}}{R}
$$


where

$$
E_{m n}=-\frac{N}{R}\left(\frac{2 m \pi}{L}\right)^{2} D_{m n}\left[\beta_{1}\left(\frac{2 m \pi}{L}\right)^{4}+\beta_{3}\left(\frac{2 m \pi}{L}\right)^{2}\left(\frac{n}{R}\right)^{2}+\beta_{5}\left(\frac{n}{R}\right)^{4}\right]^{-1} .
$$

In order to apply the Bubnov-Galerkin method, we need to verify the linearly independence of the system of functions in the (3.1).

Lemma. The system of functions

$$
\delta w_{m n}=\left(1-\cos \frac{2 m \pi x_{1}}{L}\right) \sin \frac{n x_{2}}{R} \quad(m, n=1,2, \ldots, M)
$$

is linearly independence.

Proof. Let's consider a linear combination

$$
\sum_{m=1}^{M} \sum_{n=1}^{M} \gamma_{m n} \delta w_{m n}=0 \quad \forall x_{1} \in[0, L], \forall x_{2} \in[0,2 \pi R] .
$$

Multiplying both sides of $(3.4)$ by $\sin \frac{j x_{2}}{R}(j=1,2, \ldots, M)$ and integrating the received expression with respect to $x_{2}$ on the segment $[0,2 \pi R]$, we have

$$
\int_{0}^{2 \pi R} \sum_{m=1}^{M} \sum_{n=1}^{M} \gamma_{m n} \delta w_{m n} \sin \frac{j x_{2}}{R} d x_{2}=0
$$

Since

$$
\int_{0}^{2 \pi R} \delta w_{m n} \sin \frac{j x_{2}}{R} d x_{2}= \begin{cases}0 & \text { with } n \neq j \\ \pi R\left(1-\cos \frac{2 m \pi x_{1}}{L}\right) & \text { with } n=j .\end{cases}
$$

Thus the relation (3.5) becomes

$$
\pi R \sum_{m=1}^{M} \gamma_{m j}\left(1-\cos \frac{2 m \pi x_{1}}{L}\right)=0 \quad \forall x_{1} \in[0, L], \quad \forall j=1,2, \ldots, M .
$$

For demonstrate $\gamma_{m j}=0 \quad \forall n, j=1, \ldots, m$, we choose

$$
x_{1}=\frac{L}{2}, \quad x_{1}=\frac{L}{4}, \ldots, x_{1}=\frac{L}{2 M} .
$$

Substituting in turn these values of $x_{1}$ into (3.6), we receive

$$
\gamma_{1 j}=0, \quad \gamma_{2 j}=0, \ldots, \gamma_{M j}=0 \quad \forall j=1,2, \ldots, M \text {. }
$$

This leads to $\gamma_{m j}=0 \quad \forall m, j=1,2, \ldots, M$.

This result demonstrates that the system of functions $\delta w_{m n}$ is linearly independent. So the lemma is proven. 
From the chosen system of functions, we can use the Bubnov-Galerkin method to get

$$
\begin{aligned}
& \int_{0}^{L} \int_{0}^{2 \pi R}\left\{\alpha_{1} \frac{\partial^{4} \delta w}{\partial x_{1}^{4}}+\alpha_{3} \frac{\partial^{4} \delta w}{\partial x_{1}^{2} \partial x_{2}^{2}}+\alpha_{5} \frac{\partial^{4} \delta w}{\partial x_{2}^{4}}+\frac{9}{h^{2} N}\left(p \frac{\partial^{2} \delta w}{\partial x_{1}^{2}}+q \frac{\partial^{2} \delta w}{\partial x_{2}^{2}}\right)\right. \\
& \left.-\frac{9}{h^{2} N R} \frac{\partial^{2} \varphi}{\partial x_{1}^{2}}\right\}\left(1-\cos \frac{2 i \pi x_{1}}{L}\right) \sin \frac{j x_{2}}{R} d x_{1} d x_{2}=0 \quad(i, j=1,2, \ldots, M) .
\end{aligned}
$$

For taking this integral, first of all substituting $\delta w$ and $\varphi$ represented by (3.1) and (3.2) into (3.7), afterwards integrating that received expression, we will obtain a system of linear algebraic equations with the unknowns $D_{i j}$ written in the matrix form as follows

$$
\left[a_{i j}\right]\left[D_{i j}\right]=0, \quad i, j=1,2, \ldots, M \text {. }
$$

Because of the condition on the existence of non-trivial solution i.e. $D_{i j} \neq 0$ then the determinant of the coefficients of $D_{i j}$ must be equal to zero

$$
\operatorname{det}\left[a_{i j}\right]=0, \quad i, j=1,2, \ldots, M
$$

Associating this expression with (1.3) and by using the parameter method, we can find the critical value $t^{*}$ of the loading parameter and the critical forces $p^{*}, q^{*}$.

Note that the development of the determinant (3.7a) in general case is mathematically complicated, therefore we will take the solution in the first and second approximations

a) The first approximated solution: we choose $\delta w$ and $\varphi$ in the form

$$
\begin{aligned}
\delta w & =D_{m n}\left(1-\cos \frac{2 m \pi x_{1}}{L}\right) \sin \frac{n x_{2}}{R} \\
\varphi & =-\frac{\frac{N}{R}\left(\frac{2 m \pi}{L}\right)^{2} D_{m n} \cos \frac{2 m \pi x_{1}}{L} \sin \frac{n x_{2}}{R}}{\beta_{1}\left(\frac{2 m \pi}{L}\right)^{4}+\beta_{3}\left(\frac{2 m \pi}{L}\right)^{2}\left(\frac{n}{R}\right)^{2}+\beta_{5}\left(\frac{n}{R}\right)^{4}} .
\end{aligned}
$$

Substituting $\delta w, \varphi$ into (3.7) and taking that integral, gives us

$$
\begin{aligned}
& \frac{1}{2} \pi R L\left\{\alpha_{1}\left(\frac{2 m \pi}{L}\right)^{4}+\alpha_{3}\left(\frac{2 m \pi}{L}\right)^{2}\left(\frac{n}{R}\right)^{2}+3 \alpha_{5}\left(\frac{n}{R}\right)^{4}-\frac{9}{h^{2} N}\left[p\left(\frac{2 m \pi}{L}\right)^{2}+3 q\left(\frac{n}{R}\right)^{2}\right]\right. \\
& \left.+\frac{9}{h^{2} R^{2}}\left(\frac{2 m \pi}{L}\right)^{4}\left[\beta_{1}\left(\frac{2 m \pi}{L}\right)^{4}+\beta_{3}\left(\frac{2 m \pi}{L}\right)^{2}\left(\frac{n}{R}\right)^{2}+\beta_{5}\left(\frac{n}{R}\right)^{4}\right]^{-1}\right\} D_{m n}=0 .
\end{aligned}
$$

Because of the existence of non-trivial solution i.e. $D_{m n} \neq 0$, yields a relation for finding critical loads

$$
\begin{aligned}
p\left(\frac{2 m \pi}{L}\right)^{2}+3 q\left(\frac{n}{R}\right)^{2}= & \frac{h^{2} N}{9}\left\{\alpha_{1}\left(\frac{2 m \pi}{L}\right)^{4}+\alpha_{3}\left(\frac{2 m \pi}{L}\right)^{2}\left(\frac{n}{R}\right)^{2}+3 \alpha_{5}\left(\frac{n}{R}\right)^{4}\right\} \\
& +\frac{N}{R^{2}}\left(\frac{2 m \pi}{L}\right)^{4}\left\{\beta_{1}\left(\frac{2 m \pi}{L}\right)^{4}+\beta_{3}\left(\frac{2 m \pi}{L}\right)^{2}\left(\frac{n}{R}\right)^{2}+\beta_{5}\left(\frac{n}{R}\right)^{4}\right\}^{-1}
\end{aligned}
$$


Using notations $\xi=n^{2}, \eta=\left(\frac{2 m \pi R}{L n}\right)^{2}, i=\frac{3 R}{h}$, the equation (3.8) is written in the form

$$
i^{2}=\frac{N \xi^{2}\left(\alpha_{1} \eta+\alpha_{3}+\frac{3 \alpha_{5}}{\eta}\right)\left(\beta_{1} \eta+\beta_{3}+\frac{\beta_{5}}{\eta}\right)}{\xi\left(p+\frac{3 q}{\eta}\right)\left(\beta_{1} \eta+\beta_{3}+\frac{\beta_{5}}{\eta}\right)-N} .
$$

Minimizing this relation i.e. $\frac{\partial i^{2}}{\partial \xi}=0, \frac{\partial i^{2}}{\partial \eta}=0$, after some calculations we have

$$
\begin{gathered}
\xi=\frac{2 N}{\left(p+\frac{3 q}{\eta}\right)\left(\beta_{1} \eta+\beta_{3}+\frac{\beta_{5}}{\eta}\right)} . \\
\left(\alpha_{1}-\frac{3 \alpha_{5}}{\eta^{2}}\right)\left(\beta_{1} \eta+\beta_{3}+\frac{\beta_{5}}{\eta}\right)-\left(\beta_{1}-\frac{\beta_{5}}{\eta^{2}}\right)\left(\alpha_{1} \eta+\alpha_{3}+\frac{3 \alpha_{5}}{\eta}\right)+ \\
\frac{6 q}{\eta^{2}\left(p+\frac{3 q}{\eta}\right)}\left(\alpha_{1} \eta+\alpha_{3}+\frac{3 \alpha_{5}}{\eta}\right)\left(\beta_{1} \eta+\beta_{3}+\frac{\beta_{5}}{\eta}\right)=0 .
\end{gathered}
$$

Substituting the values of $\xi$ and $\eta$ in the expressions (3.10), (3.11) into (3.9), gives us

$$
\begin{aligned}
i^{2}= & \frac{4 N^{2} \eta^{2}}{(p \eta+3 q)^{2}}\left\{\left[1-\frac{3}{4}\left(1-\frac{\phi^{\prime}}{N}\right) \frac{p^{2}}{p^{2}-p q+q^{2}}\right] \eta^{2}+\left[2-\frac{3}{2}\left(1-\frac{\phi^{\prime}}{N}\right) \frac{p q}{p^{2}-p q+q^{2}}\right] \eta\right. \\
& \left.+3\left[1-\frac{3}{4}\left(1-\frac{\phi^{\prime}}{N}\right) \frac{q^{2}}{p^{2}-p q+q^{2}}\right]\right\} \cdot\left\{\left[1+\frac{1}{4}\left(\frac{N}{\phi^{\prime}}-1\right) \frac{(2 q-p)^{2}}{p^{2}-p q+q^{2}}\right] \eta^{2}\right. \\
& \left.+\left[2+\frac{1}{2}\left(\frac{N}{\phi^{\prime}}-1\right) \frac{(2 q-p)(2 p-q)}{p^{2}-p q+q^{2}}\right] \eta+1+\frac{1}{4}\left(\frac{N}{\phi^{\prime}}-1\right) \frac{(2 p-q)^{2}}{p^{2}-p q+q^{2}}\right\}^{-1}
\end{aligned}
$$

where $\eta$ is a solution of the equation (3.11).

In order to reach a values of critical loads, we need to solve simultaneously the equation (1.3) and (3.12) by applying the loading parameter method [1]. After determining the critical value $t^{*}$, we can find the critical forces as follows

$$
p^{*}=p\left(t^{*}\right), \quad q^{*}=q\left(t^{*}\right) .
$$

Now consider an interesting case. It is a long cylindrical shell. Based on [2], we have

$$
\xi=1, \quad \eta \ll 1, \quad i^{2}=\frac{3 N \alpha_{5} \beta_{5}}{(p \eta+3 q) \beta_{5}-N \eta^{2}} .
$$

The minimization of the relation (3.13), i.e. $\frac{\partial i^{2}}{\partial \eta}=0$, yields

$$
\eta=\frac{p \beta_{5}}{2 N}=\eta^{*} .
$$


And again

$$
\left.\frac{\partial^{2} i^{2}}{\partial \eta^{2}}\right|_{\eta=\eta^{*}}=\frac{6 N^{2} \alpha_{5} \beta_{5}}{\left(3 q \beta_{5}+\frac{p^{2} \beta_{5}^{2}}{4 N}\right)^{2}} .
$$

Since $\alpha_{5}>0, \beta_{5}>0$ then $\left.\frac{\partial^{2} i^{2}}{\partial \eta^{2}}\right|_{\eta=\eta^{*}}>0$; The sufficient condition of minimum is satisfied. Taking into account $\alpha_{5}, \beta_{5}, \eta^{*}$, the relation (3.13) is written in the form

$$
i^{2}=\frac{12 N^{2}\left[1-\frac{3}{4}\left(1-\frac{\phi^{\prime}}{N}\right) \frac{q^{2}}{p^{2}-p q+q^{2}}\right]}{p^{2}\left[1+\frac{1}{4}\left(\frac{N}{\phi^{\prime}}-1\right) \frac{(2 p-q)^{2}}{p^{2}-p q+q^{2}}\right]+12 N q} .
$$

b) The second approximated solution: We take the solution as follows

$$
\begin{aligned}
\delta w & =D_{11}\left(1-\cos \frac{2 \pi x_{1}}{L}\right) \sin \frac{x_{2}}{R}+D_{21}\left(1-\cos \frac{4 \pi x_{1}}{L}\right) \sin \frac{x_{2}}{R}, \\
\varphi & =\bar{D}_{11} \cos \frac{2 \pi x_{1}}{L} \sin \frac{x_{2}}{R}+\bar{D}_{21} \cos \frac{4 \pi x_{1}}{L} \sin \frac{x_{2}}{R},
\end{aligned}
$$

where

$$
\begin{aligned}
& \bar{D}_{11}=-\frac{N}{R}\left(\frac{2 \pi}{L}\right)^{2}\left[\beta_{1}\left(\frac{2 \pi}{L}\right)^{4}+\beta_{3}\left(\frac{2 \pi}{L}\right)^{2}\left(\frac{1}{R}\right)^{2}+\beta_{5}\left(\frac{1}{R}\right)^{4}\right]^{-1} D_{11}, \\
& \bar{D}_{21}=-\frac{N}{R}\left(\frac{4 \pi}{L}\right)^{2}\left[\beta_{1}\left(\frac{4 \pi}{L}\right)^{4}+\beta_{3}\left(\frac{4 \pi}{L}\right)^{2}\left(\frac{1}{R}\right)^{2}+\beta_{5}\left(\frac{1}{R}\right)^{4}\right]^{-1} D_{21} .
\end{aligned}
$$

Substituting (3.16) into (3.7) and taking this integral, we obtain a system of two linear algebraic equations with the unknowns $D_{11}, D_{21}$. From the condition $D_{11} \neq 0, D_{21} \neq 0$ we have the relation which permits to determining the critical loads

$$
\begin{aligned}
& \left\{\left(\frac{2 \pi}{L}\right)^{4} \alpha_{1}+\left(\frac{2 \pi}{L}\right)^{2}\left(\frac{1}{R}\right)^{2} \alpha_{3}+3 \alpha_{5}\left(\frac{1}{R}\right)^{4}-\frac{9}{h^{2} N}\left[\left(\frac{2 \pi}{L}\right)^{2} p+3 q\left(\frac{1}{R}\right)^{2}\right]+\right. \\
& \left.\frac{\frac{9}{h^{2} R^{2}}\left(\frac{2 \pi}{L}\right)^{4}}{\beta_{1}\left(\frac{2 \pi}{L}\right)^{4}+\beta_{3}\left(\frac{2 \pi}{L}\right)^{2}\left(\frac{1}{R}\right)^{2}+\beta_{5}\left(\frac{1}{R}\right)^{4}}\right\} \cdot\left\{\left(\frac{4 \pi}{L}\right)^{4} \alpha_{1}+\left(\frac{4 \pi}{L}\right)^{2}\left(\frac{1}{R}\right)^{2} \alpha_{3}+\right. \\
& \left.3 \alpha_{5}\left(\frac{1}{R}\right)^{4}-\frac{9}{h^{2} N}\left[\left(\frac{4 \pi}{L}\right)^{2} p+3 q\left(\frac{1}{R}\right)^{2}\right]+\frac{\frac{9}{h^{2} R^{2}}\left(\frac{4 \pi}{L}\right)^{4}}{\beta_{1}\left(\frac{4 \pi}{L}\right)^{4}+\beta_{3}\left(\frac{4 \pi}{L}\right)^{2}\left(\frac{1}{R}\right)^{2}+\beta_{5}\left(\frac{1}{R}\right)^{4}}\right\} \\
& -\left[2\left(\frac{1}{R}\right)^{4} \alpha_{5}-\frac{18 q}{h^{2} R^{2} N}\right]^{2}=0 .
\end{aligned}
$$

\section{Some results of numerical calculation and discussion}

We consider the long cylindrical shell made of the steel $30 X \Gamma C A$ with an elastic modulus $3 G=2.6 \cdot 10^{5} \mathrm{MPa}$, an yield point $\sigma_{s}=400 \mathrm{MPa}$ (see [1]). 
The relations for determining the critical loads are given in the form

* formulae (2.12) and (1.3) for the part a) of the examples.

* Formulae (3.15) and (1.3) for the part b) of the examples.

The numerical results are realized by the MATLAB program.

Example 1. Suppose that the complex loading law is of the form

$$
p \equiv p(t)=\frac{\left(p_{0}+p_{1} t\right)^{2}}{p_{1}}, \quad q \equiv q(t)=q_{0}+q_{1} t,
$$

where $p_{0}=2 \mathrm{MPa}, p_{1}=0.1 \mathrm{MPa}, q_{0}=2 \mathrm{MPa}, q_{1}=0.1 \mathrm{MPa}$.

a) Numerical results for the simply supported cylindrical shell

Table 1

\begin{tabular}{|c|c|c|c|c|c|}
\hline$\frac{R}{h}$ & $t^{*}$ & $s \cdot 10^{3}$ & $p^{*} \mathrm{MPa}$ & $q^{*} \mathrm{MPa}$ & $\sigma_{u}^{*} \mathrm{MPa}$ \\
\hline 20 & 59.34 & 10.51 & 629.5 & 7.9 & 625.6 \\
\hline 31 & 54.81 & 5.031 & 559.6 & 7.5 & 555.9 \\
\hline 40 & 52.95 & 3.469 & 531.7 & 7.3 & 528.1 \\
\hline 50 & 51.17 & 2.469 & 506.5 & 7.1 & 502.9 \\
\hline 59 & 49.45 & 1.905 & 482.3 & 6.9 & 478.9 \\
\hline 65 & 48.10 & 1.669 & 463.8 & 6.8 & 460.4 \\
\hline 68 & 41.13 & 1.276 & 373.7 & 6.1 & 370.7 \\
\hline 77 & 28.21 & 0.7346 & 232.4 & 4.8 & 230.0 \\
\hline
\end{tabular}

b) Numerical results for the clamped cylindrical shell

Table 2

\begin{tabular}{|c|c|c|c|c|c|}
\hline$\frac{R}{h}$ & $t^{*}$ & $s \cdot 10^{3}$ & $p^{*} \mathrm{MPa}$ & $q^{*} \mathrm{MPa}$ & $\sigma_{u}^{*} \mathrm{MPa}$ \\
\hline 20 & 62.45 & 15.46 & 679.8 & 8.245 & 675.7 \\
\hline 31 & 56.47 & 6.844 & 584.7 & 7.647 & 581.0 \\
\hline 40 & 54.02 & 4.285 & 547.9 & 7.402 & 544.2 \\
\hline 50 & 51.87 & 2.821 & 516.5 & 7.187 & 512.9 \\
\hline 59 & 50.11 & 2.090 & 491.5 & 7.011 & 488.1 \\
\hline 65 & 48.62 & 1.739 & 470.9 & 6.862 & 467.5 \\
\hline 68 & 47.11 & 1.579 & 450.4 & 6.711 & 447.1 \\
\hline 71 & 36.97 & 1.087 & 324.6 & 5.697 & 321.7 \\
\hline 77 & 28.55 & 0.747 & 235.7 & 4.855 & 233.3 \\
\hline
\end{tabular}

Example 2. The complex loading law is given in the form

$$
\begin{array}{lll}
p \equiv p(t)=p_{0}+p_{1} t^{3}, & p_{0}=2 \mathrm{MPa}, & p_{1}=0.1 \mathrm{MPa} \\
q \equiv q(t)=q_{0}+q_{1} t^{2}, & q_{0}=2 \mathrm{MPa}, & q_{1}=0.1 \mathrm{MPa} .
\end{array}
$$


a) Results of numerical calculation for the simply supported cylindrical shell

Table 3

\begin{tabular}{|c|c|c|c|c|c|}
\hline$\frac{R}{h}$ & $t^{*}$ & $s \cdot 10^{3}$ & $p^{*} \mathrm{MPa}$ & $q^{*} \mathrm{MPa}$ & $\sigma_{u}^{*} \mathrm{MPa}$ \\
\hline 17 & 17.52 & 5.329 & 539.7 & 32.69 & 524.2 \\
\hline 20 & 17.27 & 3.961 & 517.0 & 31.82 & 501.9 \\
\hline 23 & 17.03 & 2.992 & 495.5 & 30.99 & 480.7 \\
\hline 25 & 16.89 & 2.563 & 484.2 & 30.54 & 469.7 \\
\hline 28 & 16.67 & 2.060 & 465.6 & 29.80 & 451.5 \\
\hline 30 & 16.49 & 1.798 & 450.3 & 29.19 & 436.4 \\
\hline 33 & 15.62 & 1.440 & 382.8 & 26.39 & 370.3 \\
\hline 35 & 14.66 & 1.173 & 316.9 & 23.49 & 305.9 \\
\hline
\end{tabular}

b) Results for the clamped cylindrical shell

Table 4

\begin{tabular}{lcccccc}
\hline$\frac{R}{h}$ & & $t^{*}$ & $s \cdot 10^{3}$ & $p^{*} \mathrm{MPa}$ & $q^{*} \mathrm{MPa}$ & $\sigma_{u}^{*} \mathrm{MPa}$ \\
\cline { 1 - 1 } & & & & & & \\
17 & & 17.60 & 5.864 & 547.4 & 32.98 & 531.7 \\
20 & 17.33 & 4.222 & 522.1 & 32.02 & 506.9 \\
23 & 17.07 & 3.165 & 499.4 & 31.14 & 484.6 \\
25 & 16.93 & 2.671 & 487.3 & 30.66 & 472.7 \\
28 & 16.71 & 2.117 & 468.2 & 29.91 & 454.0 \\
30 & 16.52 & 1.828 & 452.5 & 29.28 & 438.6 \\
33 & 15.65 & 1.446 & 385.0 & 26.48 & 372.5 \\
35 & 14.68 & 1.178 & 318.4 & 23.55 & 307.3 \\
\hline
\end{tabular}

The above results lead us to some conclusions

1. We have used the Bubnov-Galerkin method for solving the elastoplastic stability problem of the cylindrical shells with two types of various kinematic boundary conditions. In this paper, the linearly independence of the systems of functions $\delta w_{i j}$ are also investigated.

2. For long shells we have shown the necessary and sufficient conditions of minimum.

3. The more the shell is thin the more the value of critical stress intensity $\sigma_{u}^{*}$ is small (see Tables 1, 2, 3, 4).

4. The critical loads of the simply supported cylindrical shells subjected to complex loading are always smaller than critical ones when the cylindrical shells are clamped. This result corresponds to the real property of material (see Tables 1, 2, 3, 4).

5. Theory of elastoplastic processes can be applied to the stability problem of cylindrical shells when both pre-buckling and post-buckling processes are complicated.

This paper is completed with financial support from the National Basic Research Program in Natural Sciences. 


\section{References}

1. Dao Huy Bich, Theory of Elastoplastic Processes, Vietnam National University Publishing House, Hanoi, 1999 (in Vietnamese).

2. Volmir A. S., Stability of Deformable Systems, Moscow 1963 (in Russian).

3. Ogibalov P. M., Kontunov M. A., Plates and Shells, Moscow, 1969 (in Russian).

4. Dao Van Dung, Stability Problem outside Elastic Limit According to the Theory of Elastoplastic Processes, Ph. D. Thesis, Hanoi 1993 (in Vietnamese).

5. Dao Van Dung, Solving method for stability problem of elastoplastic cylindrical shells with compressible material subjected to complex loading processes, Vietnam Journal of Mechanics 23 (2) (2001) 69-86.

6. Hill $\mathrm{R}$, Plastic deformation and instability in thin-walled tuber under combined loadding: a general theory, Journal of Mech. and Phys. of Solids 47 (1999) 921-933.

Received May 23, 2003

\section{VỀ BÀI TOÁN ỔN ĐỊNH ĐÀN DÉO CƯA VỎ TRỰ TRÒN MỎNG CHỊU TẢI PHỨC TẠP VỚI CÁC ĐIỀU KIỆN BIÊN ĐỘNG HỌC KHÁC NHAU}

Bài báo trình bày bài toán ổn định của vỏ trụ chịu tác dụng đồng thời cá lực nén dọc đường sinh và áp lực ngoài. Đã xét hai dạng điều kiện biên động học là tựa bản lề và ngàm tại $x_{1}=0 ; x_{1}=L$. Sử dụng phương pháp Bubnov-Galerkin đã thiết lập được hệ thức để tìm tải tới hạn. Điều kiện đủ của cực trị cho vỏ dài đã được xem xét. Một số kết quả tính toán bằng số cũng được trình bày và thăo luận. 